\title{
Chronic Chagas disease: can prophylaxis and therapeutic vaccines crack this 'hard nut'?
}

\author{
"Although, none of the therapeutic vaccines assessed so far have \\ reached sterilizing immunity, the results obtained in terms of \\ disease control indicate that immune intervention might be used for \\ therapeutic purposes."
}

First draft submitted: 26 November 2015; Accepted for publication: 27 November 2015; Published online: 20 January 2016

Keywords: chronic Chagas disease $\bullet$ immuotherapy • vaccine

American trypanosomiasis (Chagas disease) is one of the major health problems in Latin America and has emerged as worldwide health problem in the last few years [1]. Mostly, all individuals who are infected by the protozoan Trypanosoma cruzi develop an asymptomatic acute phase; with only $1 \%$ of them experiencing nonspecific symptoms. After a variable time, about $20-30 \%$ of infected people progress to chronic Chagas disease (CCD), which is characterized by mega-syndromes involving the heart and/ or the digestive tract. These conditions are responsible for the disabilities and deaths seen among such infected individuals [2].

The underlying mechanisms for CCD imply parasite persistence with the accompanying inflammatory process, and the autoimmune response induced by $T$. cruzi [3]. Also, it has been reported that CCD might be a consequence of functional impairment of pathogen-specific T cells [4]. Despite progress in understanding the pathogenesis of CDD, we still cannot fully explain and predict why and who among the infected individuals will eventually develop symptomatic CCD.

Concerning the treatment, two molecules are currently available as anti-trypanosomal drugs: benznidazole and nifurtimox. Both have shown good efficacy during the acute phase and in the early chronic phase of CCD in children up to 15 years old (level of evidence from meta-analysis of randomized controlled trials) [5]. Nevertheless, as mentioned above, the acute phase is mostly asymptomatic and hence most of the infected individuals who will progress toward chronicity are not diagnosed. In this regard, other important questions are still unanswered; should I treat patients with CCD?, Is the anti-trypanosomal treatment effective to 'cure' individuals with CCD?

On the assumption that reduction in the parasite persistence (and the coexistent inflammatory process) by anti-trypanosomal drugs (including posconazole) might improve the clinical course of CCD, several clinical trials were performed. Unfortunately, these trials and systematic reviews on these studies failed to provide clear favorable outcomes $[5,6]$. Since none of the studies evaluated hard endpoints and the follow-up was short (up to 4 years), the BENEFIT Trial was carried out. This multicenter, randomized controlled clinical trial was designed to analyze whether benznidazole treatment is able to improve the clinical profile of individuals with Chagas' cardiomyopathy, and to promote confirmation of negativization by PCR. This represented the first study with a considerable sample size ( $\mathrm{n}=2854$ ) and a longer follow-up (median of 5 years). Despite higher rate of PCR-negative conversion in the benznidazole group, there were no difference in the primary outcomes

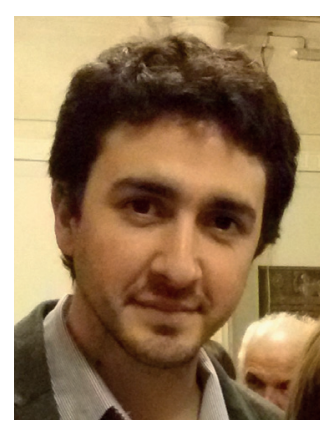

Iván Bontempi

Laboratorio de Tecnología Inmunológica, Facultad de Bioquímica y Ciencias Biológicas, Universidad Nacional del Litoral, Santa Fe, Argentina

Miguel Hernán Vicco Author for correspondence: Laboratorio de Tecnología Inmunológica, Facultad de Bioquímica y Ciencias Biológicas, Universidad Nacional del Litoral, Santa Fe, Argentina

and

Facultad de Ciencias Médicas, Universidad Nacional del Litoral, Santa Fe, Argentina Tel.: +54 03424575215 Int:125 mvicco@santafe-conicet.gov.ar Jagadeesh Bayry Institut National de la Santé et de la Recherche Médicale Unité 1138, Centre de Recherche des Cordeliers, Paris, France

Future $\because \%$ Medicine ${ }_{\text {part of }}$ 
(cardiovascular events) between placebo and benznidazole-treated groups [7]. Recently, the TRAENA trial, that aimed at assessing whether benznidazole modifies the natural evolution of asymptomatic CCD, has been concluded. The results of this study are awaited.

According to available data, there seems to be no benefit in treating CCD patients to elude disease progression. However, beside the limitations of the different studies, the lack of efficacy of benznidazole to prevent progression toward CCD could be due to inadequate treatment as suggested by the BENEFIT report. We should bear in mind that CCD might be independent of parasite persistence and that another physiopathological mechanism, that is, autoimmune response triggered by the protozoan might elicit CCD [3].

\section{"To date, promising results have been achieved in the acute phase experimental infections in mice and in the prevention of symptoms of chronic disease."}

In addition to pharmacological approach, several research groups explored the usefulness of vaccines for CCD. These explorations have been hindered, mainly due to the assumption that vaccines could trigger a severe autoimmune response. Conversely, many reports concluded that specific parasite antigens could be used without inducing autoimmune reaction [8]. In experimental animal models, although none of them were shown to be effective in preventing the infection, they were able to control the disease. Based on these results, the current vaccine research lines are currently oriented on three different aspects: design new vaccines to achieve prevention of the infection; obtain therapeutic vaccines to avoid or delay the involvement of heart and/or digestive tract in individuals with asymptomatic CCD; and obtain therapeutic vaccines to reverse tissue lesions.

Over the years, several T. cruzi vaccines employing diverse approaches were developed. These strategies include live attenuated or killed parasite immunization, purified protein immunization, recombinant protein immunization and DNA, bacteria or virus heterologous delivery antigen vaccines [8]. From the vast amount of antigens evaluated, promising results were obtained by CZ, TC24, TcG2, TcG4 and several trans-sialidase family proteins such as TS, ASP2 and TSA-1. As IFN- $\gamma$ has a protective role in Chagas disease, naked DNA vaccines and viral or bacterial vectors have been employed for the vaccination purposes to boost Th1 response [9]. These approaches have yielded promising results in terms of reducing the parasitemia, cardiac inflammation and parasite burden. These vaccines also tended to reduce the development of cardiac arrhythmias in the chronic stage. Furthermore, recent data showed a longterm efficacy of a prime-boost vaccine, capable of responding to challenge infection 4-6 months post immunization in mice [10]. Similarly, a recombinant human adenovirus vaccine conferred immune protection to the challenge of 70 days after the first prime immunization [11]. However, live recombinant virus or genetic immunization approaches have to pass numerous safety tests that will take many years or decades. Fortunately, researchers are now aware of this urgent requirement of safer vaccines for Chagas disease. For example, Dumonteil E et al., who extensively worked on DNA vaccine for Chagas disease [12], are currently focusing on attempts to reproduce the effect of DNA vaccines by immunizing with their recombinant protein counterparts formulated with alum adjuvant together with the TLR 4 agonist [13]. Some authors have already developed subunit vaccines using recombinant proteins formulated with adjuvants of new generations to induce both humoral and cellular immune responses and protection during chronic stage of $T$. cruzi infection, that is, less parasitemia, lower parasite load and fibrotic tissue [14-16]. As $\mathrm{CD} 4{ }^{+} \mathrm{CD} 25^{+} \mathrm{FoxP}^{+}$Tregs influence the clinical course of the disease, adjuvant molecules such as small molecule CCR 4 antagonists that transiently inhibit the Tregs during immunization could be explored for the vaccination to boost protective cellular and humoral responses [17].

To date, promising results have been achieved in the acute phase experimental infections in mice and in the prevention of symptoms of chronic disease. These results allow us to think that although prophylactic vaccines may not provide sterilizing immunity, they might be used to prevent the disease. Indeed this vaccine approach is being already used in other infectious diseases such as acellular pertussis vaccine. This vaccine has been widely used to prevent whooping cough and severe symptoms of the infection with high efficacy. However, it is now known that it fails to prevent colonization of the bacteria [18]. Finally, several labs are evaluating the therapeutic vaccines in chronic infection models $[10-11,13,19]$. These vaccines not only prevented but also reversed the clinical and pathological lesions as assessed by ECG, levels of transaminases or histology both in mice and dogs infected with T. cruzi. These beneficial effects have been correlated with a decrease in T. cruzi persistence in blood and tissue, a lower inflammatory and antioxidant state and antiinflammatory cytokine profile. Although, none of the therapeutic vaccines assessed so far have reached sterilizing immunity, the results obtained in terms of disease control indicate that immune intervention might be 
used for therapeutic purposes. This approach is widely investigated in HIV vaccine research, field where sterilizing immunity seems to be a chimera but where low levels of viral load have shown to avoid AIDS and virus contagion [20].

Given this background, the only effective and available weapon continues to be vector control, which was shown to decrease the incidence and prevalence of Chagas disease in endemic areas. After more than a hundred years of Carlos Chagas first description, we are still awaiting acceptable and successful therapeutic control measures for CCD. Immunotherapeutic has been considered as a possible approach over the last two

\section{References}

1 Hotez PJ, Dumonteil E, Woc-Colburn L et al. Chagas disease: "the new HIV/AIDS of the Americas". PLoS Negl. Trop. Dis. 6(5), e1498 (2012).

2 Rassi A Jr, Rassi A, Marcondes de Rezende J. American trypanosomiasis (Chagas disease). Infect. Dis. Clin. North Am. 26(2), 275-291 (2012).

3 Bonney KM, Engman DM. Autoimmune pathogenesis of Chagas heart disease: looking back, looking ahead. Am. J. Pathol. 185(6), 1537-1547 (2015).

4 Argüello RJ, Albareda MC, Alvarez MG et al. Inhibitory receptors are expressed by Trypanosoma cruzi-specific effector $\mathrm{T}$ cells and in hearts of subjects with chronic Chagas disease. PLoS ONE 7(5), e35966 (2012).

5 Villar JC, Perez JG, Cortes OL et al. Trypanocidal drugs for chronic asymptomatic Trypanosoma cruzi infection. Cochrane Database Syst. Rev. 27(5), CD003463 (2014).

6 Sguassero Y, Cuesta CB, Roberts KN et al. Course of chronic Trypanosoma cruzi infection after treatment based on parasitological and serological tests: a systematic review of follow-up studies. PLoS ONE 10(10), e0139363 (2015).

7 Morillo CA, Marin-Neto JA, Avezum A et al. Randomized trial of benznidazole for chronic Chagas' cardiomyopathy. N. Engl. J. Med. 373(14), 1295-1306 (2015).

8 Rodríguez-Morales O, Monteón-Padilla V, Carrillo-Sánchez SC et al. Experimental vaccines against Chagas disease: a journey through history. J. Immunol. Res. 2015, 489758 (2015).

9 Arce-Fonseca M, Rios-Castro M, Carrillo-Sánchez Sdel C, Martínez-Cruz M, Rodríguez-Morales O. Prophylactic and therapeutic DNA vaccines against Chagas disease. Parasit. Vectors 8(121), 121 (2015).

10 Gupta S, Garg NJ. A two-component DNA-prime/ protein-boost vaccination strategy for eliciting long-term, protective $\mathrm{T}$ cell immunity against Trypanosoma cruzi. PLoS Pathog. 11(5), e1004828 (2015).

11 Pereira IR, Vilar-Pereira G, Marques V et al. A human type 5 adenovirus-based Trypanosoma cruzi therapeutic decades and preclinical results are promising. Though we might get success in the future, for the time being CCD remains 'a hard nut to crack'.

\section{Financial \& competing interests disclosure}

The authors have no relevant affiliations or financial involvement with any organization or entity with a financial interest in or financial conflict with the subject matter or materials discussed in the manuscript. This includes employment, consultancies, honoraria, stock ownership or options, expert testimony, grants or patents received or pending, or royalties.

No writing assistance was utilized in the production of this manuscript.

vaccine re-programs immune response and reverses chronic cardiomyopathy. PLoS Pathog. 11(1), e1004594 (2015).

12 Dumonteil E, Escobedo-Ortegon J, Reyes-Rodriguez N, Arjona-Torres A, Ramirez-Sierra MJ. Immunotherapy of Trypanosoma cruzi infection with DNA vaccines in mice. Infect. Immun. 72(1), 46-53 (2004).

13 Dumonteil E, Bottazzi ME, Zhan B et al. Accelerating the development of a therapeutic vaccine for human Chagas disease: rationale and prospects. Expert Rev. Vaccines 11(9), 1043-1055 (2012).

14 Hoft DF, Eickhoff CS, Giddings OK, Vasconcelos JR, Rodrigues MM. Trans-sialidase recombinant protein mixed with $\mathrm{CpG}$ motif-containing oligodeoxynucleotide induces protective mucosal and systemic Trypanosoma cruzi immunity involving CD8 ${ }^{+} \mathrm{CTL}$ and $\mathrm{B}$ cell-mediated crosspriming. J. Immunol. 179(10), 6889-6900 (2007).

15 Giddings OK, Eickhoff CS, Sullivan NL, Hoft DF. Intranasal vaccinations with the trans-sialidase Antigen plus CpG Adjuvant induce mucosalimmunity protective against conjunctival Trypanosoma cruzi challenges. Infect. Immun. 78(3), 1333-1338 (2010).

16 Bontempi IA, Vicco MH, Cabrera G et al. Efficacy of a trans-sialidase-ISCOMATRIX subunit vaccine candidate to protect against experimental Chagas disease. Vaccine 33(10), 1274-1283 (2015).

17 Bayry J, Tartour E, Tough DF. Targeting CCR4 as an emerging strategy for cancer therapy and vaccines. Trends Pharmacol. Sci. 35(4), 163-165 (2014).

18 Warfel JM, Zimmerman LI, Merkel TJ. Acellular pertussis vaccines protect against disease but fail to prevent infection and transmission in a nonhuman primate model. Proc. Natl Acad. Sci. USA 111(2), 787-792 (2014).

19 Cerny N, Alberti AS, Bivona AE et al. Coadministration of cruzipain and GM-CSF DNAs, a new immunotherapeutic vaccine against Trypanosoma cruzi infection. Hum. Vaccin. Immunother. doi:10.1080/21645515.2015.1078044 (2015) (Epub ahead of print).

20 Esparza J. A brief history of the global effort to develop a preventive HIV vaccine. Vaccine 31(35), 3502-3518 
Editorial Bontempi, Vicco \& Bayry

\author{
(2013)." To date, promising results have been \\ achieved \\ in the acute phase experimental infections \\ in mice and in the prevention of symptoms \\ of chronic disease."
}

